\title{
Inflammation and thrombosis in cardiovascular disease
}

\author{
Prabhakara Nagareddy, PhD $^{1}$ and Susan S. Smyth, MD, PhD 1,2 \\ ${ }^{1}$ Division of Cardiovascular Medicine, The Gill Heart Institute, University of Kentucky, Lexington \\ KY \\ ${ }^{2}$ Lexington VA Medical Center, Lexington KY
}

\begin{abstract}
Purpose of the review-This article will summarize recent observations that provide mechanistic insight into the molecular and cellular links between inflammation and thrombosis in the context of cardiovascular and other thromboinflammatory disease states.
\end{abstract}

Recent findings-Several disease conditions are characterized by a thromboinflammatory state in which interactions of blood cells and components with the vascular wall perpetuate both thrombotic and inflammatory pathways. Targeting these pathways may be of benefit in inflammatory conditions and cardiovascular disease, respectively.

Summary-Ongoing clinical trials should provide additional insight into the hypothesis that the thromboinflammatory state contributes to adverse clinical outcomes.

\section{Keywords}

Inflammation; Thrombosis; Cardiovascular disease

\section{Introduction}

\begin{abstract}
Atherosclerosis involves a complex interplay between metabolic pathways governing lipid deposition, inflammatory and immune responses to oxidized lipids, and endothelial dysfunction. Myocardial infarction (MI) and stroke ensue when these processes culminate in thrombosis. Markers of inflammation, such as C-reactive protein (CRP), myeloperoxidase (MPO) and leukocyte levels are strong predictors of cardiovascular death, MI, and stroke. Similarly, individuals with chronic inflammatory conditions such as rheumatoid arthritis or systemic lupus erythematous (SLE) are at higher risk for the development and complications of atherosclerosis. This review will summarize recent observations that provide mechanistic insight into the molecular and cellular links between inflammation and thrombosis in the context of cardiovascular disease (CVD), which support the concept of a thromboinflammatory state.
\end{abstract}

Address correspondence to: Susan S. Smyth, MD, PhD, Division of Cardiovascular Medicine, The Gill Heart Institute, 255 BBRSB, 741 S. Limestone Street, Lexington, KY 40536-0200, Phone: 859-323-2274, Fax: 859-257-3235, SusanSmyth@uky.edu. 


\section{Platelet-mediated interactions}

Platelet activation results in the expression of cell surface receptors, adhesion, and small molecules that can alter the properties of leukocytes, endothelial cells, and other vascular cells [1]. Thus, both by direct contact and through the release of bioactive mediators, platelets may influence the function of surrounding cells. Activated platelets produce eicosanoids and platelet activating factor that have potent effects on inflammation and immunity. Recent work has focused on biologically active cargo released from intracellular platelet granules upon activation. Platelet dense granules store the purine nucleotides ADP and ATP, which have roles in thrombosis, inflammation, ischemia-reperfusion injury, and potentially acute lung injury [2]. Platelet release of serotonin from dense granules may modulate mitogenic and inflammatory responses and has been implicated in neutrophil rolling and adhesion to inflamed endothelium [3]. Finally, inorganic polyphosphates are major components of dense granules that are released during platelet activation; these influence coagulation and inflammation differentially based on the size of the polyphosphate polymer [4, 5]

Platelet a-granules contain adhesive proteins, coagulation, mitogenic and angiogenic factors, CXC and CC chemokines, and other factors. One of the most abundant a-granule proteins, platelet factor 4 (PF4/CXCL4) may influence both neutrophils and macrophages and has been implicated in atherosclerosis and vascular smooth muscle cell (VSMC) responses after injury. PF4 acts at least in part through Kruppel-like factor 4 to transcriptional regulate VSMC, reduce their differentiation and promote an inflammatory phenotype [6].

After translocation from a-granules to the cell surface, P-selectin binds to leukocyte PSGL-1 to initiate physical interactions between the cells. Platelet - leukocyte interactions are then stabilized by $\beta 2$-integrin-dependent events to promote efficient leukocyte recruitment at the site of vascular injury and the formation of mixed platelet-leukocyte conjugates in circulating blood (Figure 1). Platelet - leukocyte aggregates form and may predict outcomes in acute coronary syndromes (ACS) and following percutaneous coronary intervention (PCI) [7, 8]. In the setting of ACS and PCI, platelet - leukocyte aggregates correlate with the inflammatory marker C-reactive protein (CRP) and with biomarkers of myocardial necrosis, such as troponin $[9,10]$. Anti-platelet therapy, in particular with P2Y12 antagonists, has been associated with reductions in platelet-leukocyte aggregate formation. In several clinical studies, use of the P2Y12 antagonist clopidogrel was linked to reductions in P-selectin expression, whereas aspirin was not. The active metabolites of both clopidogrel and prasugrel reduce platelet - leukocyte aggregate formation in vitro and in animal models [11, 12]. Anti-platelet therapy has been variably associated with reductions in biomarkers of systemic inflammation, including decreased CRP, CD40L, and P-selectin in a variety of disease states, including: cardiovascular disease, cerebrovascular disease, diabetes, and renal transplantation [13-16]. Reduced expression of IL-1a, 2, 6, 13, 10, TNFa, TNF $\beta$, and CRP has been observed in patients after long-term treatment with clopidogrel. [17] How much of the clinical benefit of anti-platelet therapy derives from reductions in platelet-mediated inflammation is not known. 
Strategies to target directly interactions between platelets, endothelial cells, and leukocytes are the focus of ongoing studies. Based on strong data from preclinical animal models, Pselectin appears to be an attractive target to block thromboinflammatory cell interactions. The recombinant monoclonal antibody to P-selectin, inclacumab, was tested in the SELECT-ACS trial in which patients $(\mathrm{n}=544)$ presenting with ACS were randomized to receive placebo, or one of 2 doses of inclacumab ( 5 or $20 \mathrm{mg} / \mathrm{kg}$ ) administered as a single infusion [18]. In patients who underwent PCI $(n=322)$, those receiving the higher dose of inclacumab had a reduction in cardiac injury as assessed by plasma levels of troponin I and creatinine kinase MB (CK-MB) levels, although both endpoints barely missed statistical significance $(\mathrm{P}=0.05)$. There was also a corresponding decrease in soluble $\mathrm{P}$-selectin levels. The study was underpowered to detect clinical events, however, there were numerically more deaths, MIs, and strokes in the 2 groups that received inclacumab than in the placebo control. Nonetheless, the effects on cardiac biomarkers, and especially the reduction in CKMB >3 times the upper limit of normal, may portend a clinical value to P-selectin inhibition.

\section{Neutrophil-mediated interactions}

Leukocytosis, and in particular elevations in neutrophil count, is a robust predictor of increased morbidity and mortality of ischemic vascular disease. Leukocytosis has emerged as a risk factor for both short term and long term complications in studies of $>350,000$ patients. Ischemic events themselves elicit an inflammatory response and elevations in white blood count may reflect the extent of injury. However, in the CAPRIE trial of patients with recent MI, stroke or peripheral vascular disease, leukocyte counts obtained within one week prior to a recurrent ischemic event were significantly higher than those observed at baseline or in days 8-120 before an event. Additionally, the degree of accumulation of neutrophils in aspirated thrombus of patients presenting with STEMI is an independent predictor of a poor myocardial blush grade, ST-segment resolution, and left ventricular function at 6 months. [19] These findings suggest that leukocytosis may causally impact the development of ischemic events.

Several regulators of neutrophilia and monocytosis have been proposed to impact the development of CVD. Hematopoietic stem cell proliferation and mobilization result in more monocytes and neutrophils originating from the bone marrow. Additionally, stem cell mobilization to spleen can initiate extramedullary hematopoesis. High density lipoprotein and cholesterol efflux pathways can reverse stem cell mobilization in experimental models [20-22]; whereas hyperglycemia and obesity can promote monocytosis by endogenous danger signals produced by neutrophils[23]. In mice, genetic or pharmacologic approaches that alter coagulation appear to impact atherosclerosis in a neutrophil-dependent manner, and hypercoagulable states are associated with neutrophilia and neutrophil hyper-reactivity [24].

There are numerous ways in which leukocytes impact CVD. On their own, and synergistically with platelets, they generate tissue factor, microparticles, and surfaces for thrombin generation to enhance fibrin formation. Leukocyte homotypic and plateletleukocyte heterotypic aggregates may cause rheological compromise of microvasculature. Neutrophils and monocytes also promote CVD through the generation of inflammatory 
mediators, tissue infiltration, and oxidation of lipoproteins and other proteins. Neutrophil release of the serine proteases elastase and cathepsin $\mathrm{G}$ promote efficient thrombus formation in mice by stabilizing fibrin deposition, likely by proteolytic inactivation of endogenous anticoagulants. [25]

In the last several years, evidence has emerged for a key role for neutrophil release of extracellular traps (NETs) in thrombotic conditions. In response to strong stimulation, neutrophils release extrude NETs that consist of DNA and histones in a process that involves histone citrullinated by peptidylarginine deiminase 4, chromatin unwinding, breakdown of nuclear membranes and cytolysis [26]. A key function of the extracellular chromatin material is to entrap and confine microbes to promote their destruction. The chromatin fibers may also serve as a scaffold for clot formation, promote extrinsic and intrinsic activation of the coagulation cascade, and provide resistance to thrombolysis. Several reports indicate a key role for neutrophils and NETs in venous thrombosis in animal models [27-29]. Platelets can trigger NET formation and may also bind to histones to form platelet - NET attachments. Histones activate platelets through TLR-dependent mechanisms to generate the release of polyphosphates [30], which in turn amplify coagulation. NETs have been implicated in experimental transfusion related acute lung injury, where their formation appears to be triggered by activated platelets [31]. Analysis of material obtained by aspiration of coronary thrombi indicated the presence of NETs, histone H1, myeloperoxidase, and neutrophil elastase in samples obtained from fresh clot or following thrombolysis, but not in specimens of organized material. [32] NET formation may also occur in SLE and contribute to premature CVD that occurs in these individuals [33]. Taken together, these findings demonstrate that NETs may serve as a tight link between immunity and thrombosis and suggest novel therapeutic targets for thrombosis. Indeed, in animal models, DNAse1 treatment and peptidylarginine deiminase 4-deficiency dramatically protects against venous thrombosis [34].

\section{Anti-inflammatory therapy in CVD}

A number of anti-inflammatory therapies have been tested in patients with acute MI (AMI), however, as-of-yet no therapy has emerged as having a demonstrable benefit in this setting. An example is the development of the complement (C5) inhibitor pexelizumab, which reduces neutrophil count and showed promise in several preclinical studies and a phase 2 trial of individuals with AMI undergoing PCI [35], although this was not the case in a trial of AMI patients receiving thrombolysis [36]. Also, in the APEX-AMI trial, a phase 3 study of 6,000 patients, pexelizumab had no effect on 90-day mortality [37].

The JUPITER trial demonstrated that statin therapy prevented cardiovascular events in individuals with a high inflammatory burden (assessed by CRP levels $>2 \mathrm{mg} / \mathrm{L}$ ), normal LDL-cholesterol values (LDL $<130 \mathrm{mg} / \mathrm{dL}$ ) and no known CVD. In this patient population, rosuvastatin at a dose of $20 \mathrm{mg}$ daily reduced by $54 \%$ the risk of first-ever MI, stroke by $43 \%$ [38], and deep vein thrombosis or pulmonary embolism by 43\% [39]. Due to the effects of statins on cholesterol metabolism, the JUPITER trial could not definitively establish an anti-inflammatory benefit. However, the lowest clinical event rates were observed in individuals who achieved LDL-cholesterol levels $<70 \mathrm{mg} / \mathrm{dL}$ and hsCRP levels $<2 \mathrm{mg} / \mathrm{L}$. 
The effect of anti-inflammatory therapy on vascular events is currently being testing in the Canakinumab Anti-Inflammatory Thrombosis Outcomes Study (CANTOS) and the Cardiovascular Inflammation Reduction Trial (CIRT). In CANTOS, individuals with stable coronary artery disease and elevated hsCRP will be randomized to receive the interleukin- $1 \beta$ (IL-1 $\beta$ ) inhibitor canakinumab or placebo [40]. CIRT examines the effect of low-dose methotrexate in subjects with type 2 diabetes or metabolic syndrome that have had a heart attack [41]. Both trials will measure rates of recurrent myocardial infarction, stroke, and cardiovascular death. In patients with rheumatoid arthritis, evidence suggests that methotrexate treatment [42] and TNFa inhibitors [43] may improve cardiovascular outcomes. Etanercept, a recombinant fusion protein containing the human TNF receptor that scavenges $\mathrm{TNFa}$, has been investigated in congestive heart failure [44] and administered to a small number of patients with acute MI [45], though with disappointing results.

\section{Anti-thrombotic strategies in inflammation and sepsis}

Based on compelling experimental evidence that thrombosis contributes to inflammatory states, several anti-thrombotic strategies have been explored in systemic inflammatory responses and sepsis. After initial promising results in sepsis based on the results of the PROWESS trial [46], activated protein C (drotrecogin a) received FDA approval that was subsequently limited to patients at high risk of death. In the large, international PROWESSShock study of critically ill adults with septic shock, drotrecogin a did not reduce mortality at either 28 or 90 days, as compared with placebo [47] and has subsequently been removed from the market. High-dose antithrombin has not demonstrated benefit and is associated with increased bleeding in severe sepsis [48], although it may have efficacy in disseminated intravascular coagulation associated with sepsis [49]. Likewise recombinant TFPI (rTFPI, Tifacogin) failed to improve outcomes in sepsis [50]. Recombinant thrombomodulin has shown promising results in Phase 2 sepsis studies, especially among individuals with coagulopathy and respiratory or cardiac dysfunction, and a phase 3 trial is currently underway [51].

Thrombocytopenia in the setting of sepsis portends poor outcomes for reasons that are not known. Platelets express toll-like receptors (TLRs), including TLR2 and TLR4 that recognize the bacterial peptidoglycan and lipopolysaccharide (LPS), respectively [52-54]. Interactions between LPS and platelet TLR4 have been implicated in sepsis-induced thrombocytopenia, pulmonary fibrin deposition, and microvascular thrombosis in animal models[53]. Platelet activation and sequestration in pulmonary tissue is a key feature in inflammatory states and may contribute to acute lung injury and acute respiratory distress syndrome (ARDS) [55]. Antiplatelet therapy with clopidogrel or prasugel reduces LPSmediated thrombocytopenia, fibrin deposition in the lungs, and inflammatory mediator upregulation in mice $[12,53,56]$, whereas ticagrelor therapy prevents lung damage and neutrophil infiltration in the cecal ligation puncture model of sepsis in mice (content.onlinejacc.org/data/Journals/JAC/926556/00716.pdf).

Retrospective and observational clinical studies hint that anti-platelet therapy may improve outcomes in critical illness, especially in individuals at risk for acute lung injury. In a single center experience of 224 patients hospitalized with pneumonia requiring intensive care, 
those who were taking antiplatelet therapy (aspirin, clopidogrel, or ticlopidine) had shorter lengths of stay [56]. A subsequent. larger study $(n=615)$ by the same investigators documented a reduction in mortality in medical and surgical intensive care patients who were receiving aspirin or clopidgorel [57] In population-based observational studies, prehospital anti-platelet therapy was associated with reductions in indices of acute lung injury and ARDS [58-60], although, a more recent publication by one of the groups failed to confirm the benefit of aspirin [61]. The ongoing Lung Injury Prevention With Aspirin (LIPS-A) trial will test the hypothesis that early aspirin ( $325 \mathrm{mg}$ followed by $81 \mathrm{mg}$ daily) given within 12 hours of hospitalization to individuals at risk for acute lung injury will improve outcomes [62].

\section{Conclusions}

Substantial mechanistic evidence links inflammation and thrombosis. Observations in preclinical animal models indicated the existence of thromboinflammatory conditions and suggest that targeting inflammatory and thrombotic pathways may prove benefical. Ongoing clinical trials should determine the clinical efficacy of anti-inflammatory therapy in reducing the thrombotic complications of cardiovascular disease and better define the role of antithrombotic and anti-platelet therapy in inflammatory states. Another promising strategy may be to reduce leukocyte counts, rather than targeting their function. Identification of the ideal agents, their timing and duration of administration await future studies.

\section{Acknowledgments}

This work was supported in part by the National Center for Advancing Translational Sciences, through UL1TR000117 and the Heart Lung and Blood Institute through R01HL078663. The content is solely the responsibility of the authors and does not necessarily represent the official views of the NIH. This material is also based on work supported in part by resources at the Lexington VA Medical Center.

\section{References}

1. Smyth SS, McEver RP, Weyrich AS, et al. Platelet functions beyond hemostasis. Journal of thrombosis and haemostasis: JTH. 2009; 7:1759-1766. [PubMed: 19691483]

*2. Eltzschig HK, Sitkovsky MV, Robson SC. Purinergic signaling during inflammation. The New England journal of medicine. 2013; 368:1260. This article reviews the role of ATP and ADP in inflammation. [PubMed: 23534573]

*3. Duerschmied D, Suidan GL, Demers M, et al. Platelet serotonin promotes the recruitment of neutrophils to sites of acute inflammation in mice. Blood. 2013; 121:1008-1015. This work demonstrated the serotonin released from platelet dense granules promotes neutrophil rolling and recruitment along inflammed endothelium. [PubMed: 23243271]

4. Muller F, Mutch NJ, Schenk WA, et al. Platelet polyphosphates are proinflammatory and procoagulant mediators in vivo. Cell. 2009; 139:1143-1156. [PubMed: 20005807]

5. Morrissey JH, Choi SH, Smith SA. Polyphosphate: an ancient molecule that links platelets, coagulation, and inflammation. Blood. 2012; 119:5972-5979. [PubMed: 22517894]

6. Shi G, Field DJ, Long X, et al. Platelet factor 4 mediates vascular smooth muscle cell injury responses. Blood. 2013; 121:4417-4427. [PubMed: 23568488]

7. Michelson AD, Barnard MR, Krueger LA, et al. Circulating monocyte-platelet aggregates are a more sensitive marker of in vivo platelet activation than platelet surface P-selectin: studies in baboons, human coronary intervention, and human acute myocardial infarction. Circulation. 2001; 104:1533-1537. [PubMed: 11571248] 
8. Furman MI, Barnard MR, Krueger LA, et al. Circulating monocyte-platelet aggregates are an early marker of acute myocardial infarction. Journal of the American College of Cardiology. 2001; 38:1002-1006. [PubMed: 11583872]

9. Zhang SZ, Jin YP, Qin GM, Wang JH. Association of platelet-monocyte aggregates with platelet activation, systemic inflammation, and myocardial injury in patients with non-st elevation acute coronary syndromes. Clinical cardiology. 2007; 30:26-31. [PubMed: 17262778]

10. Ray MJ, Walters DL, Bett JN, et al. Platelet-monocyte aggregates predict troponin rise after percutaneous coronary intervention and are inhibited by Abciximab. International journal of cardiology. 2005; 101:249-255. [PubMed: 15882672]

11. Evangelista V, Manarini S, Dell'Elba G, et al. Clopidogrel inhibits platelet-leukocyte adhesion and platelet dependent leukocyte activation. Thrombosis and Haemostasis. 2005; 94:568-577. [PubMed: 16268474]

*12. Totani L, Dell'Elba G, Martelli N, et al. Prasugrel inhibits platelet-leukocyte interaction and reduces inflammatory markers in a model of endotoxic shock in the mouse. Thrombosis and haemostasis. 2012; 107:1130-1140. This study demonstrated that the P2Y12 antagonist prasugrel block platelet - leukocyte aggregates and inflammation following LPS administration in mice. [PubMed: 22436970]

13. Graff J, Harder S, Wahl O, et al. Anti-inflammatory effects of clopidogrel intake in renal transplant patients: Effects on platelet-leukocyte interactions, platelet CD40 ligand expression, and proinflammatory biomarkers[ast]. Clin Pharmacol Ther. 2005; 78:468-476. [PubMed: 16321613]

14. Hermann A, Rauch BH, Braun M, et al. Platelet CD40 ligand (CD40L) - subcellular localization, regulation of expression, and inhibition by clopidogrel. Platelets. 2001; 12:74-82. [PubMed: 11297035]

15. Klinkhardt U, Bauersachs R, Adams J, et al. Clopidogrel but not aspirin reduces P-selectin expression and formation of platelet-leukocyte aggregates in patients with atherosclerotic vascular disease[ast]. Clinical Pharmacology and Therapeutics. 2003; 73:232-241. [PubMed: 12621388]

16. Steinhubl SR, Badimon JJ, Bhatt DL, et al. Clinical evidence for anti-inflammatory effects of antiplatelet therapy in patients with atherothrombotic disease. Vascular Medicine. 2007; 12:113122. [PubMed: 17615799]

17. Antonino MJ, Mahla E, Bliden KP, et al. Effect of Long-Term Clopidogrel Treatment on Platelet Function and Inflammation in Patients Undergoing Coronary Arterial Stenting. The American Journal of Cardiology. 2009; 103:1546-1550. [PubMed: 19463513]

**18. Tardif JC, Tanguay JF, Wright SS, et al. Effects of the P-Selectin Antagonist Inclacumab on Myocardial Damage After Percutaneous Coronary Intervention for Non-ST-Segment Elevation Myocardial Infarction: Results of the SELECT-ACS Trial. Journal of the American College of Cardiology. 2013; 61:2048-2055. This study demonstrated a benefit of inclacmab, a recombinant monoclonal antibody to P-selectin, on biomakers of cardiac injury in patients with acute coronary syndrome who underwent coronary intervention. [PubMed: 23500230]

19. Arakawa K, Yasuda S, Hao H, et al. Significant association between neutrophil aggregation in aspirated thrombus and myocardial damage in patients with ST-segment elevation acute myocardial infarction. Circulation journal: official journal of the Japanese Circulation Society. 2009; 73:139-144. [PubMed: 19047776]

20. Yvan-Charvet L, Pagler T, Gautier EL, et al. ATP-binding cassette transporters and HDL suppress hematopoietic stem cell proliferation. Science. 2010; 328:1689-1693. [PubMed: 20488992]

*21. Tall AR, Yvan-Charvet L, Westerterp M, Murphy AJ. Cholesterol efflux: a novel regulator of myelopoiesis and atherogenesis. Arteriosclerosis, thrombosis, and vascular biology. 2012; 32:2547-2552. This article describes pathways for cholesterol efflux that regulate hematopoeitic stem cell proliferation and extramedullary hematopoiesis.

**22. Westerterp M, Gourion-Arsiquaud S, Murphy AJ, et al. Regulation of hematopoietic stem and progenitor cell mobilization by cholesterol efflux pathways. Cell stem cell. 2012; 11:195-206. This work demonstrated a role for ABCA1, ABCG1, and HDL-cholesterol in attenuating hematopoetic stem cell mobilization and extramedullary hematopoiesis. [PubMed: 22862945]

**23. Nagareddy PR, Murphy AJ, Stirzaker RA, et al. Hyperglycemia promotes myelopoiesis and impairs the resolution of atherosclerosis. Cell metabolism. 2013; 17:695-708. This work demonstrated that neutrophilia and monocytosis in diabetice mice is the result of neutrophil 
production of S100A8/S100A9 that acts on the receptor for advanced glycation end products on common myeloid progenitor cells to enhance myelopoiesis and thereby promote atherosclerosis. [PubMed: 23663738]

*24. Borissoff JI, Otten JJ, Heeneman S, et al. Genetic and pharmacological modifications of thrombin formation in apolipoprotein e-deficient mice determine atherosclerosis severity and atherothrombosis onset in a neutrophil-dependent manner. PloS one. 2013; 8:e55784. This work demonstrated that the hypercoagulable state associated with thrombomodulin deficiency promotes neutrophilia and atherosclerosis that can be reversed by the direct thrombin inhibitor dabigatran. [PubMed: 23409043]

25. Massberg S, Grahl L, von Bruehl ML, et al. Reciprocal coupling of coagulation and innate immunity via neutrophil serine proteases. Nature medicine. 2010; 16:887-896.

*26. Fuchs TA, Brill A, Wagner DD. Neutrophil extracellular trap (NET) impact on deep vein thrombosis. Arteriosclerosis, thrombosis, and vascular biology. 2012; 32:1777-1783. This work discusses the evidence for NETosis in venous thrombus formation.

**27. von Bruhl ML, Stark K, Steinhart A, et al. Monocytes, neutrophils, and platelets cooperate to initiate and propagate venous thrombosis in mice in vivo. The Journal of experimental medicine. 2012; 209:819-835. This work identifies cross talk between monocytes, neutrophils, and platelets responsible for the initiation and amplification of venous thrombosis using intravital microscopy. The authors identify key roles for leukocyte derived tissue factor, neutrophil release of chromatin material, and GPIb-mediated platelet adhesion. [PubMed: 22451716]

*28. Darbousset R, Thomas GM, Mezouar S, et al. Tissue factor-positive neutrophils bind to injured endothelial wall and initiate thrombus formation. Blood. 2012; 120:2133-2143. This study demonstrated an essential role for neutrophils in tissue factor generation, fibrin formation, and platelet activation. [PubMed: 22837532]

*29. Brill A, Fuchs TA, Savchenko AS, et al. Neutrophil extracellular traps promote deep vein thrombosis in mice. Journal of thrombosis and haemostasis: JTH. 2012; 10:136-144. The authors demonstrate an increase in extracellular DNA following experimental venous thrombus formation and reduced clot burden with DNAse1 therapy. [PubMed: 22044575]

30. Semeraro F, Ammollo CT, Morrissey JH, et al. Extracellular histones promote thrombin generation through platelet-dependent mechanisms: involvement of platelet TLR2 and TLR4. Blood. 2011; 118:1952-1961. [PubMed: 21673343]

**31. Caudrillier A, Kessenbrock K, Gilliss BM, et al. Platelets induce neutrophil extracellular traps in transfusion-related acute lung injury. The Journal of clinical investigation. 2012; 122:2661-2671. In an animal model of TRALI, NETs appear in lung and circulating in a neutrophil and plateletdependent manner and are also observed the lungs and plasma of humans with TRALI. [PubMed: 22684106]

*32. de Boer OJ, Li X, Teeling P, et al. Neutrophils, neutrophil extracellular traps and interleukin-17 associate with the organisation of thrombi in acute myocardial infarction. Thrombosis and haemostasis. 2013; 109:290-297. Neutrophils, NETs and IL17A and IL17F were demonstrated in coronary thrombosuction specimens obtained from patients after acute myocardial infarction. [PubMed: 23238559]

**33. Knight JS, Zhao W, Luo W, et al. Peptidylarginine deiminase inhibition is immunomodulatory and vasculoprotective in murine lupus. The Journal of clinical investigation. 2013 NET formation was observed in a murine model of lupus and an inhibitor of peptidylarginine deiminases, which prevents citrullination of histones and therefore NET formation, reduced lupus-like disease activity, improved endothelial function, and attenuated the prothrombotic phenotype.

**34. Martinod K, Demers M, Fuchs TA, et al. Neutrophil histone modification by peptidylarginine deiminase 4 is critical for deep vein thrombosis in mice. Proceedings of the National Academy of Sciences of the United States of America. 2013; 110:8674-8679. Peptidylarginine deiminase 4 (PAD4)-deficient mice are dramatically protected from thrombus after inferior vena cava stenosis. PAD4 on hematopoietic cells is sufficient to attenuate thrombosis. [PubMed: 23650392]

35. Granger CB, Mahaffey KW, Weaver WD, et al. Pexelizumab, an anti-C5 complement antibody, as adjunctive therapy to primary percutaneous coronary intervention in acute myocardial infarction: the COMplement inhibition in Myocardial infarction treated with Angioplasty (COMMA) trial. Circulation. 2003; 108:1184-1190. [PubMed: 12925454] 
36. Mahaffey KW, Granger CB, Nicolau JC, et al. Effect of pexelizumab, an anti-C5 complement antibody, as adjunctive therapy to fibrinolysis in acute myocardial infarction: the COMPlement inhibition in myocardial infarction treated with thromboLYtics (COMPLY) trial. Circulation. 2003; 108:1176-1183. [PubMed: 12925455]

37. Mehta RH, Starr AZ, Lopes RD, et al. Incidence of and outcomes associated with ventricular tachycardia or fibrillation in patients undergoing primary percutaneous coronary intervention. JAMA: the journal of the American Medical Association. 2009; 301:1779-1789. [PubMed: 19417195]

38. Ridker PM, Danielson E, Fonseca FA, et al. Rosuvastatin to prevent vascular events in men and women with elevated C-reactive protein. The New England journal of medicine. 2008; 359:21952207. [PubMed: 18997196]

39. Glynn RJ, Danielson E, Fonseca FA, et al. A randomized trial of rosuvastatin in the prevention of venous thromboembolism. The New England journal of medicine. 2009; 360:1851-1861. [PubMed: 19329822]

40. Ridker PM, Thuren T, Zalewski A, Libby P. Interleukin-1beta inhibition and the prevention of recurrent cardiovascular events: rationale and design of the Canakinumab Anti-inflammatory Thrombosis Outcomes Study (CANTOS). American heart journal. 2011; 162:597-605. [PubMed: 21982649]

41. Ridker PM. Testing the inflammatory hypothesis of atherothrombosis: scientific rationale for the cardiovascular inflammation reduction trial (CIRT). Journal of thrombosis and haemostasis: JTH. 2009; 7 (Suppl 1):332-339. [PubMed: 19630828]

42. Pieringer H, Pichler M, Pohanka E, Hoppe UC. Will Antirheumatic Treatment Improve Cardiovascular Outcomes in Patients with Rheumatoid Arthritis? Current pharmaceutical design. 2013

43. Naranjo A, Sokka T, Descalzo MA, et al. Cardiovascular disease in patients with rheumatoid arthritis: results from the QUEST-RA study. Arthritis research \& therapy. 2008; 10:R30. [PubMed: 18325087]

44. Mann DL, McMurray JJ, Packer M, et al. Targeted anticytokine therapy in patients with chronic heart failure: results of the Randomized Etanercept Worldwide Evaluation (RENEWAL). Circulation. 2004; 109:1594-1602. [PubMed: 15023878]

*45. Padfield GJ, Din JN, Koushiappi E, et al. Cardiovascular effects of tumour necrosis factor alpha antagonism in patients with acute myocardial infarction: a first in human study. Heart. 2013 This trial investigated the effect of the TNF alpha scavenger etanercept on biomarkers of inflammation in patients with acute myocardial infarction. Twenty-four hours after the infusion, a reduction in neutrophil count and IL-6 levels and an increased in platelet - monocyte aggregates was observed.

46. Bernard GR, Vincent JL, Laterre PF, et al. Efficacy and safety of recombinant human activated protein C for severe sepsis. The New England journal of medicine. 2001; 344:699-709. [PubMed: 11236773]

*47. Ranieri VM, Thompson BT, Barie PS, et al. Drotrecogin alfa (activated) in adults with septic shock. The New England journal of medicine. 2012; 366:2055-2064. The PROWESS-Shock trial randomized over 6000 individuals with severe sepsis to receive activated protein $\mathrm{C}$ (dotrecogin alpha) or placebo. No benefit was observed on clinical outcomes. The fundings of this study resulted in removal of dotrecogin alpha from the market. [PubMed: 22616830]

48. Warren BL, Eid A, Singer P, et al. Caring for the critically ill patient. High-dose antithrombin III in severe sepsis: a randomized controlled trial. JAMA: the journal of the American Medical Association. 2001; 286:1869-1878. [PubMed: 11597289]

*49. Iba T, Saito D, Wada H, Asakura H. Efficacy and bleeding risk of antithrombin supplementation in septic disseminated intravascular coagulation: a prospective multicenter survey. Thrombosis research. 2012; 130:e129-133. In an observational study, patients with DIC in the setting of sepsis and reduced antithrombin levels who received antithrombin at a dose of $3000 \mathrm{IU} /$ day showed significantly improved survival. [PubMed: 22542365]

50. Abraham E, Reinhart K, Opal S, et al. Efficacy and safety of tifacogin (recombinant tissue factor pathway inhibitor) in severe sepsis: a randomized controlled trial. JAMA: the journal of the American Medical Association. 2003; 290:238-247. [PubMed: 12851279] 
*51. Iba T, Nagaoka I, Boulat M. The anticoagulant therapy for sepsis-associated disseminated intravascular coagulation. Thrombosis research. 2013; 131:383-389. This article discusses different options for anticoagulant therapy in DIC associated with sepsis. [PubMed: 23566533]

52. Zhang G, Han J, Welch EJ, et al. Lipopolysaccharide stimulates platelet secretion and potentiates platelet aggregation via TLR4/MyD88 and the cGMP-dependent protein kinase pathway. Journal of immunology. 2009; 182:7997-8004.

53. Aslam R, Speck ER, Kim M, et al. Platelet Toll-like receptor expression modulates lipopolysaccharide-induced thrombocytopenia and tumor necrosis factor-alpha production in vivo. Blood. 2006; 107:637-641. [PubMed: 16179373]

54. Cognasse F, Hamzeh H, Chavarin P, et al. Evidence of Toll-like receptor molecules on human platelets. Immunology and cell biology. 2005; 83:196-198. [PubMed: 15748217]

*55. Weyrich AS, Zimmerman GA. Platelets in lung biology. Annual review of physiology. 2013; 75:569-591. This work describes the mounting evidence for a role for platelets in lung biology and as mediators of acute lung injury.

56. Winning J, Reichel J, Eisenhut Y, et al. Anti-platelet drugs and outcome in severe infection: clinical impact and underlying mechanisms. Platelets. 2009; 20:50-57. [PubMed: 19172522]

57. Winning J, Neumann J, Kohl M, et al. Antiplatelet drugs and outcome in mixed admissions to an intensive care unit. Critical Care Medicine. 2010; 38:32-37. [PubMed: 19770746]

58. Erlich JM, Talmor DS, Cartin-Ceba R, et al. Prehospitalization antiplatelet therapy is associated with a reduced incidence of acute lung injury: a population-based cohort study. Chest. 2011; 139:289-295. [PubMed: 20688925]

59. Gross AK, Dunn SP, Feola DJ, et al. Clopidogrel treatment and the incidence and severity of community acquired pneumonia in a cohort study and meta-analysis of antiplatelet therapy in pneumonia and critical illness. Journal of thrombosis and thrombolysis. 2013; 35:147-154. This study examined the association of prescriptions for clopdigrel and clinical outcomes in patients in a large Medicaid database who were hospitalized for pneumonia. A meta-analysis of observations studies suggested a benefit of anti-platelet therapy with outcomes in critical illness and lung injury. [PubMed: 23124575]

60. O’Neal HR Jr, Koyama T, Koehler EA, et al. Prehospital statin and aspirin use and the prevalence of severe sepsis and acute lung injury/acute respiratory distress syndrome. Critical care medicine. 2011; 39:1343-1350. [PubMed: 21336116]

61. Kor DJ, Erlich J, Gong MN, et al. Association of prehospitalization aspirin therapy and acute lung injury: results of a multicenter international observational study of at-risk patients. Critical care medicine. 2011; 39:2393-2400. [PubMed: 21725238]

62. Kor DJ, Talmor DS, Banner-Goodspeed VM, et al. Lung Injury Prevention with Aspirin (LIPS-A): a protocol for a multicentre randomised clinical trial in medical patients at high risk of acute lung injury. BMJ open. 2012; 2 


\section{Key Points}

- Emerging evidence suggests the existence of thromboinflammatory disease states in which platelets and leukocytes interact and generate novel mediators of inflammation and thrombosis

- A recent trial targeting P-selectin in acute myocardial infarction holds promise for reduction in ischemic damage during coronary interventions

- Anti-platelet therapy has been shown to variably reduce systemic markers of inflammation and is being studied for clinical efficacy in ongoing trials

- Ongoing clinical trials are evaluating the benefit of anti-inflammatory therapy in CVD and strategies aimed at lowering leukocyte count may prove to be a viable method of targeting inflammation and thrombosis 


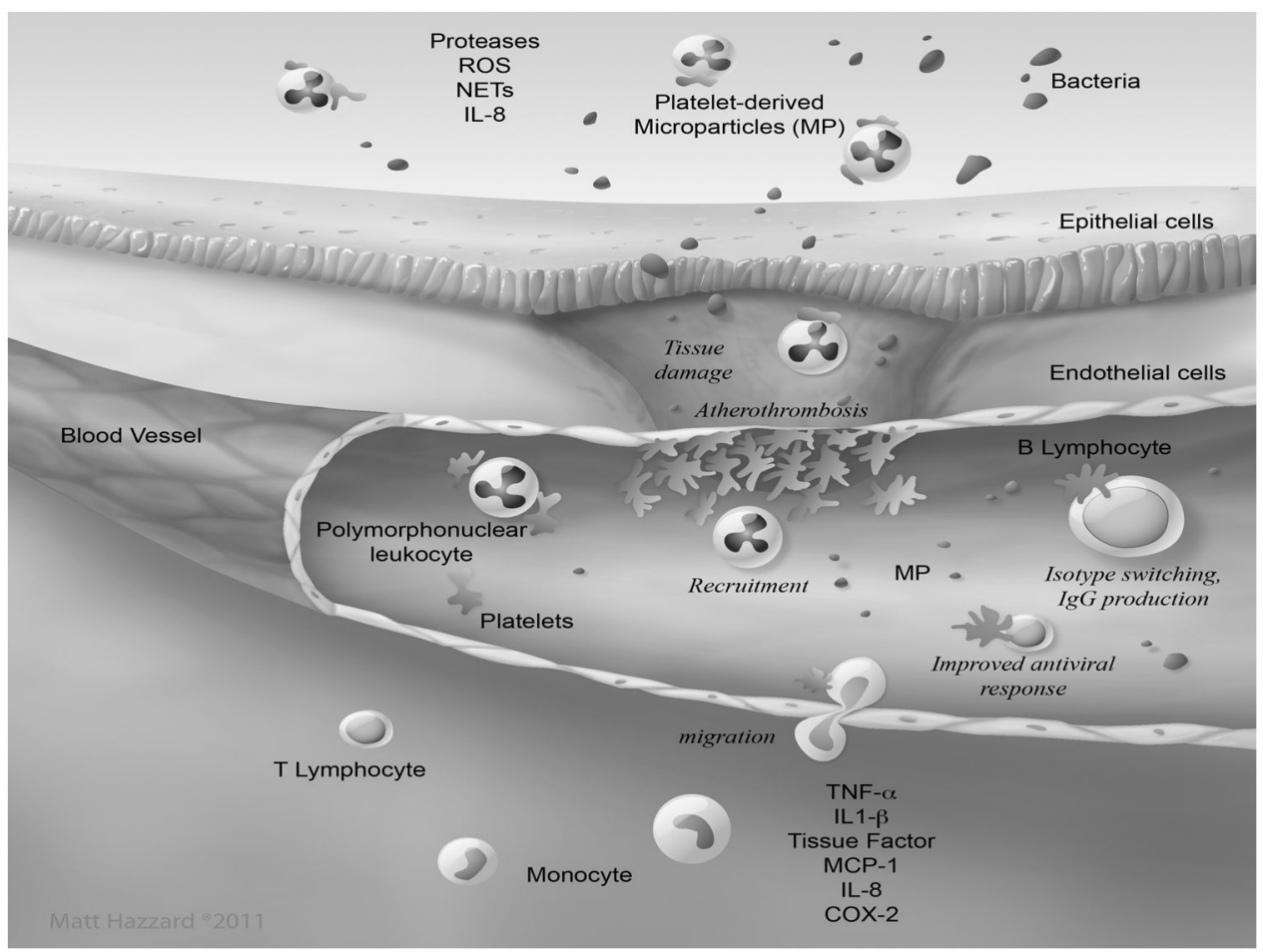

Figure 1. Contribution of platelet-leukocyte interactions to a thromboinflammatory state Platelets are activated at the site of endothelial damage or in the microcirculation of infected/inflamed tissue. Activated platelets bind leukocytes to form heterotypic complexes, and communicate signals that result in a variety of specific responses. Platelets mediate the recruitment of leukocytes at the site of atherosclerosis or thrombus formation, as well as in inflamed tissue. In some cases, these interactions mediate platelet-leukocyte co-migration across the mucosal epithelium. In this way, platelets contribute to the promotion of inflammatory reactions, which, when not controlled, can exacerbate tissue damage. Platelets might support lymphocyte homing in peripheral lymph nodes, stimulate isotype switching and production of IgG by B lymphocytes, and might help lymphocyte responses to viruses and neutrophil response to bacteria. In this way, platelets contribute to host defense. Illustration by Matt Hazzard, University of Kentucky, Information Technology. 